\section{ACIDENTES DE TRABALHO GRAVES EM ADOLESCENTES}

\section{SERIOUS WORK ACCIDENTS IN TEENAGERS}

Andréia de Souza Santos ${ }^{1, *} /$ Grasielle da Silva Santos ${ }^{1}$ / Maiane Silva Pereira ${ }^{1} /$ Marcela Andrade Rios ${ }^{2}$ / Raissa Neyla da Silva Domingues Nogueira ${ }^{1} /$ Tânia Teixeira de Figueiredo ${ }^{1}$

\section{INTRODUÇÃO}

De acordo com a Organização Mundial da Saúde (OMS), adolescência é o período que se estende 10 aos 19 anos de idade. Já o Estatuto da Criança e do Adolescente (ECA) define como adolescentes pessoas com idade de 12 a 18 anos. (BRASIL, 1990).

A adolescência é caracterizada por uma fase de intensas descobertas e modificações, principalmente físicas e psicológicas, já que nessa fase ocorre a transição da infância para a vida adulta. É comum nessa época que os adolescentes exerçam atividades laborais, visto como algo naturalizado principalmente em famílias de baixo nível econômico. Entretanto, quando estes desempenham atividades que os exponham a riscos contra sua saúde, gera uma repercussão em torno do assunto, visto que atividades desenvolvidas sem treinamentos podem desencadear acidentes e possíveis sequelas decorrentes do mesmo (MUNIZ et al., 2019).

A utilização da força de trabalho infanto-juvenil é um tema de grande relevância, pois abrange diversos aspectos como culturais, econômicos, psicológicos, físicos e outros. Ainda assim, desperta atenção sobre a necessidade de erradicação dessa prática quando a mesma é desenvolvida em ambientes degradantes e insalubres (PIMENTA et al., 2013).

O Estatuto da Criança e do Adolescente (ECA) proíbe expressamente qualquer tipo de trabalho para menores de quatorze anos de idade, sendo permitidas atividades entre 14 e 16 anos, desde que, em atividades não perigosas e condizentes com o seu desenvolvimento físico e psicológico em regime de aprendiz (OIT, 2009).

\section{RESUMO}

Objetivo: descrever os acidentes de trabalho em adolescentes, no período de 2007 a 2018, no estado da Bahia. Materiais e Métodos: trata-se de um estudo descritivo, com dados disponibilizados pelo Sistema de Informação de Agravos de Notificação, as variáveis analisadas foram divididas em variáveis sociodemográficas e relacionadas ao acidente, foram incluídas as notificações ocorridas no período entre 2007 a 2018, que se tratavam de acidentes de trabalho graves em adolescentes de 12 a 18 anos de idade. Resultados: entre 2007 a 2018 foram identificados 885 casos de acidentes de trabalho envolvendo adolescentes, no estado da Bahia. $\mathrm{O}$ ano de $2018 \mathrm{com}(14 \%$; $\mathrm{n}=124)$ obteve o maior quantitativo de acidentes de trabalho notificados, o sexo masculino foi mais acometido com $(91,3 \%$; $\mathrm{n}=808)$ casos, a maior parte dos acidentados tinham 18 anos idade $(38,6 \%, \mathrm{n}=342)$, da raça parda $(52,4 \%, \mathrm{n}=464)$, residente na zona urbana $(69.2 \%, \mathrm{n}=612)$, à macrorregião que contabilizou mais registros foi a Leste $(35,3 \% ; \mathrm{n}=312)$, o município com maior prevalência foi Salvador $(18,8 \%, \mathrm{n}=165)$, a maioria dos adolescentes sofreram acidentes de trabalho (AT) típico (69,5\%; $n=615)$. Conclusão: Assim, os resultados encontrados no presente estudo apontam para a necessidade de criação de políticas públicas para proteção desses adolescentes.

Palavras-chave: Adolescente. Trabalho infantil. Acidentes de trabalho.

\section{ABSTRACT}

Objective: to describe occupational accidents in adolescents, from 2007 to 2018, in the state of Bahia. Materials and methods: this is a descriptive study, with available data by the Notification Recording Information System, as analyzed variables divided into sociodemographic variables and related to accidents, notifications from 2007 to 2018 were included, that these were serious work accidents in adolescents aged 12 to 18 years. Results: between 2007 and 2018, 885 cases of occupational accidents involving adolescents were identified, in the state of Bahia. The year 2018 with $(14 \% ; n=124)$ obtained the highest number of reported work accidents, the male sex was more affected with $(91.3 \% ; \mathrm{n}=808)$ cases, most of the victims were 18 years old $(38.6 \%, \mathrm{n}=342)$, brown $(52.4 \%, \mathrm{n}=464)$, resident in the urban area $(69.2 \%, n=612)$, the macro-region that accounted for more records was the East $(35.3 \% ; n=312)$, the municipality with the highest prevalence was Salvador $(18.8 \%, \mathrm{n}=165)$, the majority of adolescents suffered typical occupational accidents (TA) $(69.5 \% ; n=615)$. Conclusion: Thus, the results found in the present study point to the need to create public policies to protect these adolescents.

Keywords: Adolescent. Child labor. Accidents at work.

Submetido em: 08 de nov. 2019

Aceito em: 14 de fev. 2020

${ }^{1}$ Universidade do Estado da Bahia - UNEB, Guanambi, Bahia - Brasil.

${ }^{2}$ Universidade Estadual do Sudoeste da Bahia, Jequié, Bahia - Brasil.

${ }^{*}$ E-mail para correspondência: andreiaenf264@gmail.com

Rev. ComCiência - jun. 2020, vol. 5, no. 6, p. 95-99/ doi: 10.36112/ issn2595-1890.v5.i6.p95-99 
Contudo, pode-se observar que muitos jovens desempenham suas atividades na informalidade sem nenhuma supervisão e orientação, arriscando a sua segurança e podendo gerar danos muitas vezes irreparáveis a sua saúde (PIMENTA et al., 2013.

Desse modo caracteriza acidentes de trabalho graves aquele que ocasiona mutilação, física ou funcional, e que leva à lesão que implique em comprometimento extremamente sério, preocupante, e que pode ter implicações graves ou fatais (BRASIL,2006). Assim, o objetivo desse trabalho é descrever os acidentes de trabalho em adolescentes, no período de 2007 a 2018, no estado da Bahia.

\section{MATERIAIS E MÉTODOS}

Trata-se de um estudo descritivo, realizado com base em dados referentes aos casos notificados de acidentes de trabalho graves em adolescentes no estado da Bahia, registrados no Sistema de Informação de Agravos de Notificação (SINAN), entre os anos de 2007 a 2018. Para construção do estudo foram utilizados dados fornecidos pela Diretoria de Vigilância Epidemiológica (DIVEP - SESAB).

Foram incluídas as notificações ocorridas no período compreendido entre 2007 a 2018, as quais se tratavam de notificação de acidente de trabalho grave em adolescentes de 12 a 18 anos de idade.

As variáveis analisadas foram divididas em variáveis sociodemográficas e relacionadas ao acidente, sendo as sociodemográficas: sexo, idade, raça/cor, zona de residência e região do estado. $\mathrm{E}$ as relacionadas ao acidente: local do acidente, tipo do acidente, diagnóstico de acidente e de lesão, evolução do caso, setor de atividade econômica e ano.
Os dados foram tabulados e analisados com auxílio do Microsoft Office Excel, com cálculos das frequências absolutas e relativas, o que possibilitou a construção de tabelas.

Por se tratar de um estudo com dados secundários e de domínio público, não houve submissão ao Comitê de Ética em Pesquisa.

\section{RESULTADOS E DISCUSSÃO}

O presente estudo contou com dados do período entre 2007 a 2018, na qual foram identificados 885 casos de acidentes de trabalho envolvendo adolescentes, no estado da Bahia.

$O$ ano de 2018 (14\%; $n=124)$ obteve o maior quantitativo de acidentes de trabalho notificados, seguido pelos anos de 2014 (12,2\%, $\mathrm{n}=108)$, $2013(11,5 \%, \mathrm{n}=102), 2016(11,4 \%$, $\mathrm{n}=101), 2014$ e 2017 (11\%, n=97) com o mesmo quantitativo de acidentes. Enquanto isso, em 2007 (1,5\%; $\mathrm{n}=13)$ foram registradas apenas 13 notificações, seguido pelos anos de 2008 (1,6\%, $\mathrm{n}=14), 2009 \quad(3,7 \%, \mathrm{n}=33)$, $2012(7,2 \%, n=64), 2011(7,3 \%, n=65)$ e $2010(7,6 \%, n=67)$.

De acordo com dados visualizados na tabela 1 , verificou-se que, grandes partes das notificações foram do sexo masculino (91,3\%; $\mathrm{n}=808)$, enquanto que $8,7 \%(n=77)$ foram para o sexo feminino. Isso pode ser justificado diante dos processos históricos e culturais da sociedade com relação ao gênero, em que a exposição do sexo masculino com relação a esses acontecimentos é maiores, além da maior liberdade que é dada ao homem do que a mulher quanto à procura precoce por trabalho (SOUZA, 2005).
Tabela 1 - Características sociodemográficas segundo sexo, idade, raça/cor, zona de residência e macro região, na Bahia entre os anos de 2007 a 2018.

\begin{tabular}{|c|c|c|}
\hline Variáveis & $\mathbf{n}$ & $\%$ \\
\hline \multicolumn{3}{|l|}{ SEXO } \\
\hline Masculino & 808 & 91,3 \\
\hline Feminino & 77 & 8,7 \\
\hline \multicolumn{3}{|l|}{ IDADE } \\
\hline 12 anos & 9 & 1 \\
\hline 13 anos & 21 & 2,4 \\
\hline 14 anos & 36 & 4,1 \\
\hline 15 anos & 71 & 8,0 \\
\hline 16 anos & 147 & 16,6 \\
\hline 17 anos & 259 & 29,3 \\
\hline 18 anos & 342 & 38,6 \\
\hline \multicolumn{3}{|l|}{ RAÇA/COR } \\
\hline Ignorado/branco & 188 & 21,2 \\
\hline Branca & 89 & 10,1 \\
\hline Preta & 139 & 15,7 \\
\hline Amarela & 4 & 0,5 \\
\hline Parda & 464 & 52,4 \\
\hline Indígena & 1 & 0,1 \\
\hline \multicolumn{3}{|l|}{ ZONA DE RESIDÊNCIA } \\
\hline Ignorado/branco & 38 & 4,3 \\
\hline Urbana & 612 & 69,2 \\
\hline Rural & 234 & 26,4 \\
\hline Periurbana & 1 & 0,1 \\
\hline \multicolumn{3}{|l|}{ MACRORREGIÃO } \\
\hline Centro-Leste & 111 & 12,5 \\
\hline Centro-Norte & 12 & 1,4 \\
\hline Extremo Sul & 90 & 10,2 \\
\hline Leste & 312 & 35,3 \\
\hline Nordeste & 21 & 2,4 \\
\hline Norte & 26 & 2,9 \\
\hline Oeste & 64 & 7,2 \\
\hline Sudoeste & 87 & 9,8 \\
\hline Sul & 162 & 18,3 \\
\hline TOTAL & 885 & 100 \\
\hline
\end{tabular}

Fonte: SINAN - Sistema de Informações de Agravos de Notificação, 2019

A faixa etária de acidentes varia muito, sendo a idade de 12 anos, a fase de menor acometimento $(1 \%, \mathrm{n}=9)$. Enquanto as faixas etárias que registraram os maiores números de acidentes foram de 17 anos $(29,3 \%, \mathrm{n}=259)$ e de 18 anos $(38,6 \%, n=342)$. O trabalho no período da adolescência ocorre como um ponto complexo e plurifacetado que deve iniciar analisando as principais justificativas que levam a entrada de adolescentes no mercado de trabalho, e que pelo menos nas grandes cidades um dos motivos é a necessidade em contribuir com a renda familiar.

No Brasil, os jovens chegam prematuramente no trabalho, muitos ainda na infância, colocando-os em risco a saúde e o desenvolvimento pessoal (COSTA et al., 2008). Dessa forma, o labor precocemente é confi- 
gurado como um grave problema de Saúde Pública no Brasil, sendo também um dos problemas sociais mais importantes para os indivíduos desta faixa etária. Assim, há a necessidade de entender a complexidade e os riscos que podem comprometer a saúde dos jovens trabalhadores (SANTOS et al., 2009).

Com relação à raça/cor, a de maior incidência foi a parda com $54,4 \% \quad(n=464)$, seguida de branco/ignorado com 21,2\% $(n=188)$. Contudo, com muitos dados subregistrados, observa uma falha por parte do serviço de saúde, partindo dos profissionais que não realizam os devidos registros das informações, sendo extremamente importante a sua execução de forma adequada, pois os mesmos servem de subsídios para melhora da assistência de saúde e para compreender mais como que é o perfil de acidentes envolvendo os adolescentes.

Após dados sub-registrados, tem-se a cor preta com $15,7 \% \quad(n=$ 139), e indígena, a de menor notificação com $0,1 \%(n=1)$. Em um estudo realizado na cidade de Salvador, BA, com relação a óbitos por causas externas no período de 1998 a 2003, mostrou que entre os segmentos de pele raça/cor analisados, a grande perda de anos potenciais de vida ocorreu com indivíduos do sexo masculino e com os que se apresentavam raça/cor parda ou preta. Assim, a variável raça/cor possui uma aproximação com níveis socioeconômicos, de forma que a contribuição de fatores nos diferenciais achados é considerável (ARAÚJO et al., 2009).

Quanto à macrorregião, a que contabilizou maior número de registros foi a Leste, $(35,3 \% ; n=312)$ seguida da macrorregião Sul $(18,3 \%$; $=162)$, e a que registrou um menor número foi a macrorregião Centro-Norte $\quad(1,4 \%$; $\mathrm{n}=12$ ). Quanto ao município residente por macrorregião, o município de Salvador $(18,8 \%, \mathrm{n}=165)$ na macrorregião Leste apresentou o maior número de notificações, seguido por Feira de Santana $(4,7 \%, n=41)$ na região Centro-Leste e por Itabuna $(3,9 \%, n=34)$ na macrorregião Sul. A maior prevalência de acidentes encontra-se em zonas urbanas e na região leste. $\mathrm{O}$ município de Feira de Santana localizado na extensão Centro-Leste apresentou a segunda maior incidência do estado, em contraste com sua área de localização que apontou uma das maiores incidências coletados no território do estado baiano. O contingente populacional de cada cidade pode explicar este achado (PIMENTA; FREITAS; MENDES, 2013).

As menores taxas de ocorrências de acidentes de trabalhos em adolescentes na Bahia advieram da região Centro-Norte. As regiões com maior poder aquisitivo como a extensão leste obteve maiores resultados, sendo Salvador a maior incidente de todo o estado, bem como uma das contribuições que podem levar a isso são as condições econômicas podendo interferir significativamente com os resultados.

As condições precárias de trabalho e o trabalho infanto-juvenil nessas regiões é uma situação preocupante, pois estas condições e exposição desta faixa etária colocam em risco a saúde destes, a associação entre a ocorrência de acidentes de trabalho na adolescência e o uso de equipamentos de segurança individuais, devido a insalubridade do ambiente e a falta de segurança (PIMENTA; FREITAS: MENDES, 2013).
Tabela 2 - Características do acidente de trabalho segundo tipo do acidente, diagnóstico de acidente, diagnóstico de lesão e evolução do caso, na Bahia entre os anos de 2007 a 2018.

\begin{tabular}{|c|c|c|}
\hline Variáveis & $\mathbf{n}$ & $\%$ \\
\hline \multicolumn{3}{|l|}{ TIPO DE ACIDENTE } \\
\hline Ignorado/branco & 152 & 17,2 \\
\hline Típico & 615 & 69,5 \\
\hline Trajeto & 118 & 13,3 \\
\hline \multicolumn{3}{|l|}{ DIAGNÓSTICO DE ACIDENTE } \\
\hline Acidente de trabalho grave & 258 & 29,2 \\
\hline Contato com outras & 85 & 9,7 \\
\hline $\begin{array}{l}\text { Exposição a outras forças } \\
\text { mecânicas inanimadas e as } \\
\text { não especificadas }\end{array}$ & 42 & 4,7 \\
\hline $\begin{array}{l}\text { Circunstâncias relativas a } \\
\text { condições do modo de vida }\end{array}$ & 36 & 4,1 \\
\hline $\begin{array}{l}\text { Motociclista traumatizado em } \\
\text { outros acidentes de transporte }\end{array}$ & 29 & 3,3 \\
\hline $\begin{array}{l}\text { Contato com ferramentas ma- } \\
\text { nuais sem motor }\end{array}$ & 29 & 3,3 \\
\hline $\begin{array}{l}\text { Contato com faca, espada e } \\
\text { punhal. }\end{array}$ & 18 & 2,0 \\
\hline Outros & 388 & 43,8 \\
\hline \multicolumn{3}{|l|}{ DIAGNÓSTICO DE LESÃO } \\
\hline Acidente de trabalho grave & 165 & 18,6 \\
\hline $\begin{array}{l}\text { Ferimento do punho e da } \\
\text { mão, parte não especificada. }\end{array}$ & 69 & 7,7 \\
\hline $\begin{array}{l}\text { Ferimento de dedo(s) sem le- } \\
\text { são da unha }\end{array}$ & 29 & 3,2 \\
\hline $\begin{array}{l}\text { Circunstâncias relativas a } \\
\text { condições do modo de vida }\end{array}$ & 23 & 2,5 \\
\hline Ferimento do punho e da mão & 21 & 2,3 \\
\hline Fratura de dedos & 19 & 2,1 \\
\hline Outros & 559 & 63,6 \\
\hline \multicolumn{3}{|l|}{ EVOLUÇÃO } \\
\hline Ignorado/branco & 174 & 19,7 \\
\hline Cura & 232 & 26,2 \\
\hline Incapacidade temporária & 297 & 33,6 \\
\hline $\begin{array}{l}\text { Incapacidade parcial perma- } \\
\text { nente }\end{array}$ & 139 & 15,7 \\
\hline $\begin{array}{l}\text { Incapacidade total permanen- } \\
\text { te }\end{array}$ & 6 & 0,7 \\
\hline Óbito por acidente & 18 & 2,0 \\
\hline Óbito por outras causas & 2 & 0,2 \\
\hline Outra & 17 & 1,9 \\
\hline TOTAL & 885 & 100 \\
\hline
\end{tabular}

Fonte: SINAN - Sistema de Informações de Agravos de Notificação, 2019.

De acordo com os tipos de acidentes os registros foram baseados em três pontos, acidente típico, de trajeto ou ignorado/branco. Conforme a legislação brasileira os acidentes que ocorrem do percurso de casa para o trabalho e do trabalho para casa, são considerados acidentes de trajeto. Vale ressaltar que os incidentes em serviços da empresa em ambientes urbanos ou rurais também são ponderados como de trajetos, tendo como justificativa a exposição dos adolescentes a esses fenômenos por extrapolação dos serviços para recintos externos. Os aciden- 
tes típicos representam $69,5 \%$ dos ocorridos, os quais são eventos frequentes entre os jovens em consequência maior percentual de casos (SANTOS et al., 2009). Os descritos como ignorado/branco apresentou uma porcentagem de $17,2 \%$, sobressaindo aos de trajeto.

Em diagnóstico de acidentes os dados apresentados são referentes aos tipos, circunstâncias e objetos que provocou o dano. A maior taxa foi descrita como "Outros" a um percentual de $43,8 \%$, e o menor foi de $2,0 \%$ em ocorrências com objetos perfuro cortantes, "Contato com faca, punhal, espada". Os acidentes graves representam o segundo mais intercorrente. Outra especificidade nos diagnósticos de acidentes são as intercorrências relacionadas à "Exposição a outras forças mecânicas inanimadas e às não especificadas" retratando uma proporção de 4,7\%, enquanto "Circunstâncias relativas a condições do modo de vida" e "Motociclista traumatizado em outros acidentes de transporte" apontou o percentual de $4,1 \%$ e $3,3 \%$ respectivamente. São configurados como uma representação da violência social contra os jovens acidentes provenientes de condições e situações de trabalho, pelo fato de causar danos irreparáveis, temporários ou até mesmo a perda (SANTOS et al., 2009).

Quanto aos diagnósticos de lesão são registrados os tipos de lesão que acometem os jovens acidentados. Foi notificado com mais da metade $63,6 \%(n=559)$ correspondendo a outros tipos de diagnósticos, seguido por acidente de trabalho grave com $18,6 \%$ $(n=165)$ e ferimento do punho e da mão, com parte não especificada com $7,7 \% \quad(n=69)$. Esses ferimentos em mão e punho podem ser decorrentes de uso de objetos que provoquem lesões quando usados de forma inadequada, ou em uso apressado, como por exemplo, ao manuseio de uma faca. Contudo, pode verificar que a não utilização de equipamentos de proteção é considerados como um fator de risco importante para a ocorrência desses eventos. (SANTOS et al. 2009)

Em relação às consequências $33,6 \%$ ( $\mathrm{n}=297)$ evoluíram com incapacidade temporária causada pelo AT, cerca de $15,7 \%(n=139)$ tiveram incapacidade parcial permanente e $2,0 \%$ $(\mathrm{n}=18)$ vieram a óbito por acidente. Esses acidentes de trabalho que afetam jovens podem ser considerados como a ponta do iceberg, no qual consegue identificar a verdadeira condição de exploração destes trabalhadores precoces. Assim, estas ocorrências podem demonstrar condições laborais precárias e arriscadas que esses jovens vivenciam e que os expõem a riscos. (PIMENTA; FREITAS; MENDES, 2013)

Vale destacar ainda o grande quantitativo de casos de evolução que não foram registrados, apresentando 19,7\% $(n=174)$ dos dados. Quanto aos registros de acidentes por setor de atividade econômica, predominam dados ignorados $(n=818,92,3 \%)$, seguido por transporte, armazenagem e correio $(n=25,2,8 \%)$. Artes, cultura, esporte, recreação; atividades financeiras; água, esgoto, gestão de resíduos sólidos foram os setores que apresentaram o menor número de registros $(0,1 \%, n=1)$. Diante disso subentende que provavelmente as notificações não foram realizadas corretamente, o que dificulta uma avaliação mais sucinta sobre os dados apresentados.

\section{CONCLUSÃO}

A pesquisa caracterizou os casos notificados de acidentes de trabalho graves em adolescentes no estado da Bahia, entre os anos de 2007 a 2018, segundo características sociodemográficas e relacionadas ao acidente. Assim os dados obtidos revelam que a maior prevalência de acidentes foi no sexo masculino, na idade de 18 anos, na raça/cor parda, na zona de residência urbana, na macrorregião leste no município de Salvador, sendo o ano 2018 responsável pelo maior percentual de notificações.

Quanto ao tipo do acidente, o típico teve maior incidência, com diagnóstico de acidente, para acidente de trabalho grave, diagnóstico de lesão, para outros, seguido de acidente de trabalho grave, cuja evolução do caso progrediu para incapacidade temporária e quanto ao setor de atividade econômica, houve o predomínio da categoria ignorados, seguido pelo setor de transporte, armazenagem e correio.

Assim, os resultados encontrados no presente estudo apontam para a necessidade de criação de políticas públicas para proteção desses adolescentes, uma vez que estes se encontram tangenciados pelas estatísticas oficiais, e acaba sendo inseridos na atividade laborativa precocemente o que pode prejudicar sua saúde bem como o processo de escolarização e socialização dos mesmos.

Nesse contexto, faz-se imprescindível a realização de mais pesquisas que visem discutir os acidentes de trabalho graves em adolescentes, e as informações obtidas por meio das pesquisas servem de base para subsidiar a criação de políticas que realmente beneficie esses adolescentes, pontuamos como limitação deste estudo o fato do mesmo ser transversal não proporcionando um acompanhamento dos casos notificados. 


\section{REFERÊNCIAS}

ARAÚJO, E.M. et al. Diferenciais de raça/cor da pele em anos potenciais de vida perdidos por causas externas. Rev Saúde Pública, v. 43, n.3, p. 405-12, 2009.

BRASIL. Lei no 8069, de 13 de julho de 1990. Dispõe sobre o Estatuto da Criança e do Adolescente e dá outras providências. Disponível em: <http://www.planalto.gov.br/ccivil_03/ leis/18069.htm>. Acesso em: 10 out 2019

IBGE. Instituto Brasileiro de Geografia e Estatística. Censo 2010: Mais de 3 milhões de crianças e adolescentes trabalhavam no Brasil. Rio de Janeiro : IBGE., 2012. Disponível em: $<$ https://censo2010.ibge.gov.br/noticia scen-

so.html? view=noticia\&id=3\&idnoticia $=2155 \&$ busca $=1 \& \mathrm{t}=$ censo-2010-mais3-milhoes-criancas-adolescentestrabalhavam-brasil >. Acesso em: 10 out 2019

COSTA, C.P.M. et al. A associação entre a ocorrência de acidentes de trabalho na adolescência e o uso de equipamentos de segurança. Rev Adolescência e Saúde. v. 5, n. 3, 2008.

MUNIZ, L.A et al . Accidentes en el Trabajo: percepción del adolescente. Enfermería Actual de Costa Rica, n. 36, p. 7791, 2019.

OIT. Organização Internacional do Trabalho. Boas práticas do setor saúde para a erradicação do trabalho infantil. Brasília (DF): OIT; 2009. Disponível em:

<https://www.ilo.org/wcmsp5/groups/ public/---americas/---ro-lima/---ilobrasi-

lia/documents/publication/wcms_2335

85.pdf >. Acesso em: 10 out 2019

PIMENTA, A.A. et al. Acidentes de trabalho ocorridos entre adolescentes. Texto Contexto Enfermagem. v.22, n.2, p. 279-84, 2013.
SANTOS, M.E.A. et al. Trabalho precoce e acidentes ocupacionais na adolescência. Escola Anna Nery Revista de Enfermagem. v.4, n.13, p.824-32, 2009.

SOUZA, E.R. Masculinidade e violência no Brasil: contribuições para a reflexão no campo da saúde. Rev Ciência \& Saúde Coletiva, v.10, n.1, p.59-70, 2005. 